\title{
Dose uniformity of loteprednol etabonate ophthalmic gel $(0.5 \%)$ compared with branded and generic prednisolone acetate ophthalmic suspension (I\%)
}

\author{
This article was published in the following Dove Press journal: \\ Clinical Ophthalmology \\ 9 December 2013 \\ Number of times this article has been viewed
}

\section{Zora T Marlowe \\ Stephen R Davio \\ Pharmaceutical Product Development, Global Pharmaceutical Research and Development, Bausch and Lomb, Inc, Rochester, NY, USA}

Introduction: Loteprednol etabonate (LE) ophthalmic gel $0.5 \%\left(\right.$ Lotemax $^{\circledR}$ ) is a new polycarbophil-based, nonsettling topical ophthalmic formulation. The formulation is a semisolid gel at rest and a shear thinning fluid when expressed through a dropper tip. The present study was undertaken to determine how the nonsettling character of LE ophthalmic gel affects dose uniformity. Prednisolone acetate ophthalmic suspension 1\% (Pred Forte ${ }^{\circledR}$ ) and a generic prednisolone acetate suspension $1 \%$ were used as comparators.

Methods: Drug concentrations of LE ophthalmic gel, Pred Forte, and a generic prednisolone acetate suspension were determined following simulated dosing - consisting of 2 drops, expressed four times daily for 2 weeks, with bottles that were shaken or not shaken immediately prior to expressing the drops. Drug concentrations were determined using a reverse-phase high-performance liquid chromatography (HPLC) method and reported as a percentage of the declared (labeled) concentration. Comparative kinetics of drug particle sedimentation were also determined for each formulation, using dispersion analysis under gravity.

Results: Mean drug concentrations in drops of all three formulations were within a few percentage points of the declared concentration when the bottles were shaken for 5 seconds prior to dispensing. Only LE ophthalmic gel showed consistent and on-target concentrations when the bottles were unshaken prior to dispensing, with a mean (standard deviation [SD]) percent declared concentration of $102 \%(1.92 \%)$ over the 2 -week dosing regimen. Drug concentrations for the branded and generic prednisolone acetate suspensions following expression from unshaken bottles were highly variable (overall relative SDs of $16.8 \%$ and $20.3 \%$, respectively), with mean concentrations for both falling significantly below the declared concentration for drops expressed at the beginning of the 2 -week dosing regimen and significantly above the declared concentration for drops expressed near the end of the dosing regimen. Dispersion analysis at $120 \times \mathrm{g}$ showed no drug particle sedimentation for LE ophthalmic gel over the 24-hour testing period, whereas the prednisolone acetate suspensions settled in less than 6 hours.

Conclusion: LE ophthalmic gel $0.5 \%$ provided consistent dose uniformity at the declared concentration whether or not the bottle was shaken prior to dispensing, whereas Pred Forte and the generic prednisolone acetate required shaking to provide consistent drug concentrations. LE ophthalmic gel may be beneficial to patients because it eliminates the potential impact on the clinical response of both under- and overdosing.

Keywords: dose uniformity, Lotemax, Pred Forte, nonsettling, ophthalmic formulation, simulated dosing
Correspondence: Zora T Marlowe Pharmaceutical Product Development, Global Pharmaceutical R\&D, I 400 North Goodman Street, Rochester, NY 14609, USA

$\mathrm{Tel}+$ I 585338606 I

Fax +I 5853380179

Email zora.marlowe@bausch.com submit your manuscript $\mid$ www.dovepress.com Dovepress 


\section{Introduction}

Loteprednol etabonate (LE) ophthalmic gel 0.5\% (Lotemax $^{\circledR}$; Bausch \& Lomb Incorporated, Rochester, NY, USA) is a new formulation of LE approved by the US Food and Drug Administration (FDA), in 2012, for the treatment of postoperative inflammation and pain following ocular surgery. ${ }^{1}$ LE ophthalmic gel demonstrates unique rheologic properties among pharmaceutical ocular corticosteroids. ${ }^{2}$ LE gel is a semisolid under resting conditions, as demonstrated by an infinitely high viscosity at low shear stress. Yet the viscosity of the product decreases rapidly with increasing shear stress. Thus, the formulation converts to a fluid and is easily expressed as a liquid drop by the shear forces created by expression of a drop from a normal dropper bottle. After expression of a drop, the formulation returns to a semisolid in less than 1 second as it returns to a resting state. ${ }^{2}$ However, upon instillation onto the eye, the formulation loses its gel structure permanently as the polycarbophil polymer interacts with electrolytes in tears. ${ }^{2}$ The net effect of this behavior is that suspended LE drug particles do not settle in the bottle yet the formulation is easily expressed as a liquid drop and converts to a liquid on the eye, causing little, if any, temporary blurring.

A nonsettling formulation should not require shaking to disperse drug particles uniformly. A "no-shake" formulation is potentially advantageous over formulations that require shaking for the simple reason that patients' shaking behavior is a major source of variability in the amount of drug dosed. Apt et al reported that nearly two-thirds of patients did not shake their ophthalmic corticosteroid suspensions before administering a dose, despite the instructions to shake that were clearly marked on the label. ${ }^{3}$ For those patients who do shake the product, it is normally not possible to determine whether the shaking is adequate in intensity and/or duration to render the product uniform because the bottles containing ophthalmic products are typically opaque.

The purpose of this study was to determine the impact of the rheologic properties of LE ophthalmic gel $0.5 \%$ on dose uniformity. This was accomplished by determining drug concentrations in expressed drops throughout a simulated dosing regimen, for products both shaken and not shaken prior to expression of a "dose." The simulated dosing regimen consisted of the expression of 2 drops LE gel, four times daily (QID) over a period of 2 weeks, the recommended dosage and duration of treatment for patients with pain and inflammation following ophthalmic surgery. ${ }^{1}$ Two prednisolone acetate suspension products, Pred Forte ${ }^{\circledR}$ (Allergan, Inc., Irvine, CA, USA ) and a generic prednisolone acetate ophthalmic suspension (1\%), were included in the study as comparators. These prednisolone acetate products are conventional suspension products (ie, not gels) and carry instructions for shaking prior to administering a dose, on the label. ${ }^{4}$ In addition to the dose uniformity study, the nonsettling characteristics of LE ophthalmic gel were confirmed under accelerated conditions with a dispersion analysis performed under centrifugation $(120 \times g)$.

\section{Materials and methods Materials}

Commercially available lots of LE ophthalmic gel $0.5 \%$, branded prednisolone acetate ophthalmic suspension $1 \%$, and a generic prednisolone acetate ophthalmic suspension 1\% (Pacific Pharma, Inc, Irvine, CA, USA) were evaluated in this study. All three corticosteroid formulations were stored at room temperature, in their original containers $(10 \mathrm{~mL}$ bottles containing $5 \mathrm{~mL}$ of product), until use. Reference material of prednisolone acetate was purchased from USP (Lot J, catalog number 1556008; Rockville, MD, USA), and for LE, was purchased from ZaCh System SpA (Lot B; Bresso, Italy). High-performance liquid chromatography (HPLC)-grade acetonitrile and glacial acetic acid were obtained from Thermo Fisher Scientific Inc (Waltham, MA, USA), and 99.8\% unstabilized tetrahydrofuran was obtained from Alfa Aesar (Ward Hill, MA, USA). High purity deionized water (resistivity of $18.2 \mathrm{M} \Omega \cdot \mathrm{cm}$ at $25^{\circ} \mathrm{C}$ ) and 0.45 micron nylon syringe filters were obtained from EMD Millipore (Billerica, MA, USA). Synthetic optically clear polycarbonate cells (LUM rectangular synthetic cells), used in the dispersion analysis experiment, were obtained from L.U.M. GmbH (Berlin, Germany).

\section{Methods \\ Dose uniformity study}

Commercial bottles of LE ophthalmic gel, Pred Forte, and generic prednisolone acetate (two of each) were stored upright for a minimum of 12 hours prior to the start of the study. To simulate the recommended dosing of these products, 2 drops were expressed from each commercial bottles QID at 4 to 6 hour intervals for 14 days or until no product was left in the bottle. To evaluate the effect of shaking versus no shaking on dose uniformity of expressed drops, one of the bottles of each product (the same bottle each time) was shaken for 5 seconds by hand immediately prior to the drop expression, at each time point. The same analyst shook the designated bottles throughout the study to avoid introducing variability due to individual differences in shaking. All drops were dispensed into tared glass vials, capped, and stored at room temperature until analysis. 
Concentrations of LE and prednisolone acetate in the expressed drops were determined using a reverse-phase HPLC method. Samples were diluted with $1 \mathrm{~mL}$ of highpurity water and $2 \mathrm{~mL}$ of a 1:1 solution of tetrahydrofuran: acetonitrile. LE gel samples were further filtered through a 0.45 nylon syringe filter (Millex -HN; EMD Millipore, Billerica, MA, USA) prior to analysis. The HPLC system consisted of an 1200 Series HPLC, Model G1311A (Agilent Technologies Inc, Santa Clara, CA, USA) coupled with a 1200 series photodiode array detector (Agilent Technologies Inc), set at $246 \mathrm{~nm}$. Samples ( $5 \mu \mathrm{L})$ were injected on an Alltima $^{\mathrm{TM}}$ Phenyl, $5 \mu \mathrm{m}, 4.6 \times 150 \mathrm{~mm}$ HPLC column (Grace Davison Discovery Science, Deerfield, IL, USA) and eluted using gradient elution with 50\% Solvent B (acetonitrile):50\% Solvent A ( $0.5 \%$ glacial acetic acid in water) at T0 to $100 \%$ $B$ in 10 minutes, at a flow rate of $2 \mathrm{~mL} / \mathrm{min}$. The column temperature was held at $25^{\circ} \mathrm{C}$. Calibration curves for $\mathrm{LE}$ and prednisolone acetate were generated over a concentration range of 0.09 to $0.5 \mathrm{mg} / \mathrm{g}$ and 0.2 to $0.6 \mathrm{mg} / \mathrm{g}$, respectively and were found to be linear $\left(R^{2}=0.999\right.$ for both). Drop samples from all four doses dispensed on days $0,5,10$, and 15 were analyzed, whereas only two doses were analyzed on the interim days (the second and fourth dose). All samples analyzed fell within the calibration curve. Sample drug concentrations were reported as a percent of declared concentration, after adjusting for dilution factors and drop weight.

\section{Dispersion analysis experiment}

To assess the rate of settling of the drug particles in each product, sedimentation was measured directly, using a dispersion/stability analyzer (LUMiSizer ${ }^{\circledR}$, Model LS 611; L.U.M. GmbH). The LUMiSizer is a combination centrifuge and percent-transmittance spectrophotometer. Commercial bottles of LE ophthalmic gel, Pred Forte, and generic prednisolone acetate were shaken for 5 seconds before transfer of $0.4 \mathrm{~mL}$ samples to LUMiSizer cells. The LUMiSizer cells were then placed within the LUMiSizer and centrifuged at 1,000 rpm (equivalent to $118-140 \times g$ ) for 24 hours. Sedimentation rate plots were obtained using the LUMiSizer's integration (clarification) module, which calculates a normalized integral of the percent transmittance raw data at each time point. In addition, photographs were taken of the samples in their cells immediately before centrifugation and at the end of the 24-hour centrifugation, with the use of an independent camera (Powershot SX500 IS, Canon U.S.A Inc., Melville, NY, USA).

\section{Results}

Drug concentrations, reported as a percent of declared concentration, in 2-drop samples of LE ophthalmic gel $0.5 \%$, Pred Forte, and the generic prednisolone acetate ophthalmic suspension (1\%) following the simulated QID dosing for 2 weeks are shown in Figures 1 and 2. Figure 1 shows the drug concentration results from bottles shaken vigorously for 5 seconds prior to expression of each drop sample. Drug concentrations for all three products were consistent and within a few percentage points of their declared concentrations on all simulated dosing days, at all dosing time points, with a mean (standard deviation [SD]) percent declared concentration of $102 \%$ (1.43\%), 103\% (1.09\%), and 103\% $(1.36 \%)$ for the LE ophthalmic gel, Pred Forte, and generic

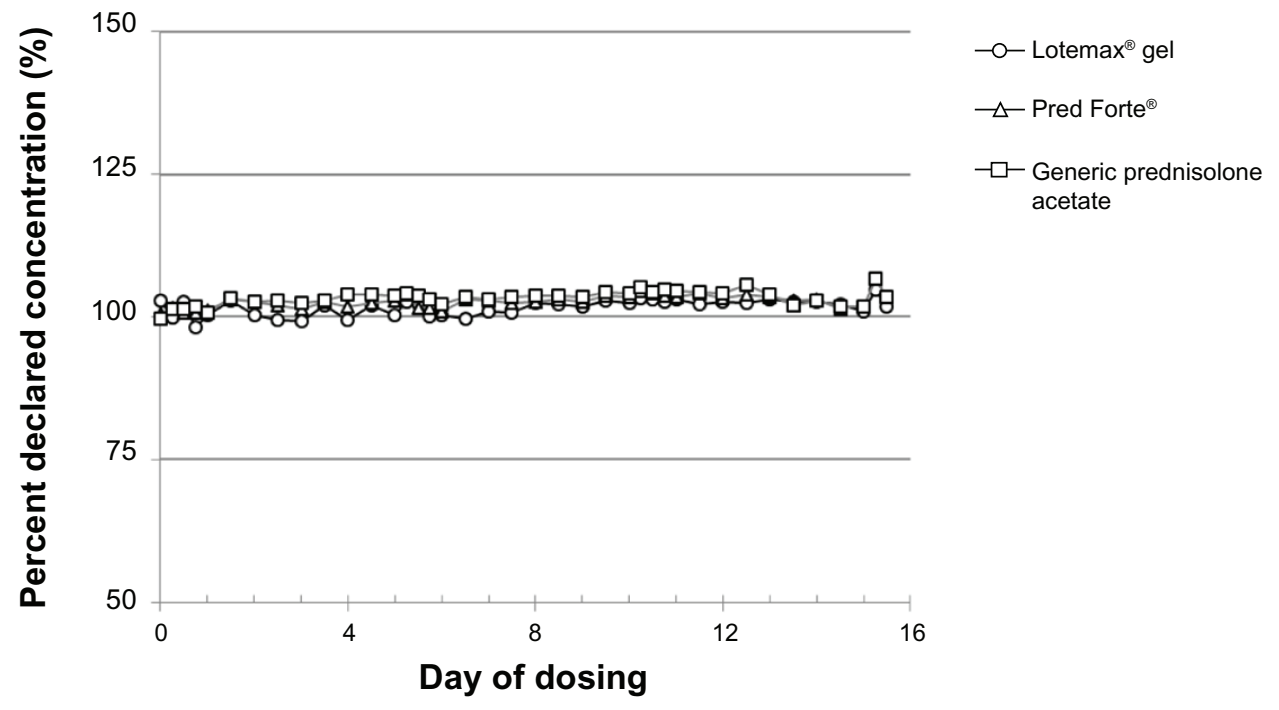

Figure I Drug concentrations in drops of Lotemax ${ }^{\circledR}$ ophthalmic gel $0.5 \%$, Pred Forte ${ }^{\circledR}$, and the generic prednisolone acetate suspension I\%, when shaken immediately prior to simulated dosing.

Notes: Lotemax ${ }^{\circledR}$, Bausch \& Lomb Incorporated, Rochester, NY, USA; Pred Forte ${ }^{\circledR}$, Allergan, Inc., Irvine, CA, USA. 


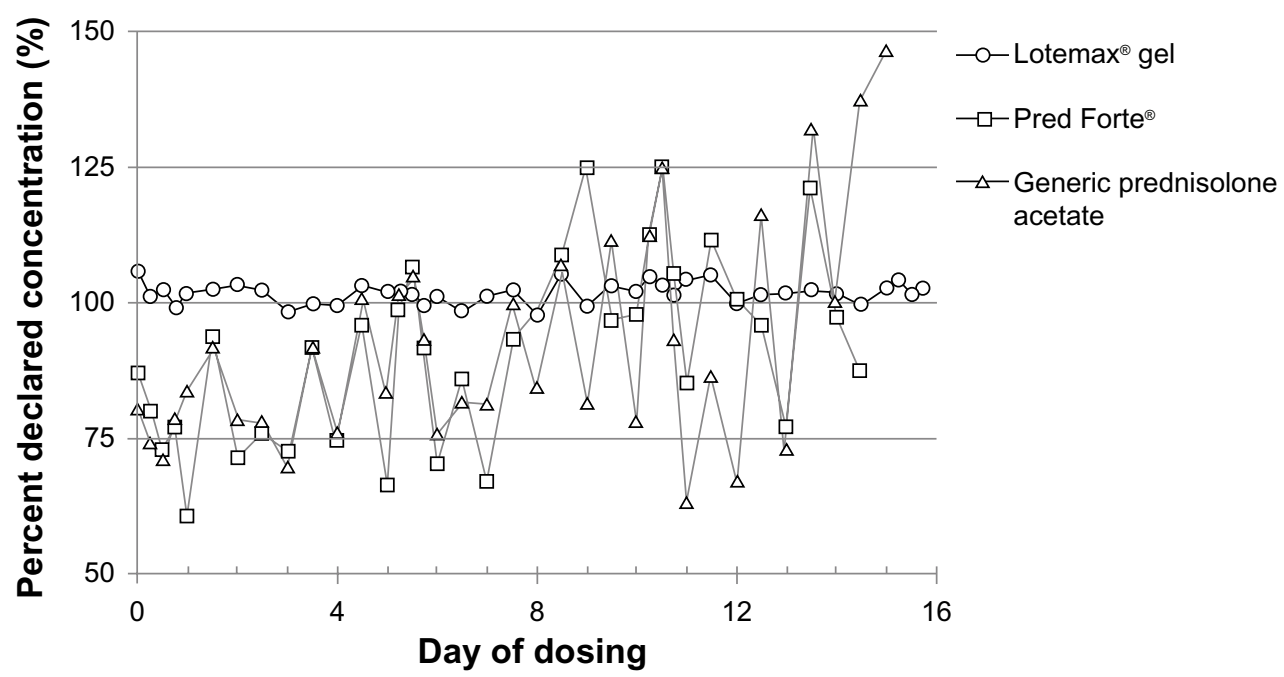

Figure 2 Drug concentrations in drops of Lotemax ${ }^{\circledR}$ ophthalmic gel $0.5 \%$, Pred Forte ${ }^{\circledR}$, and generic prednisolone acetate suspension $1 \%$, when not shaken prior to the simulated dosing.

Notes: Lotemax ${ }^{\circledR}$, Bausch \& Lomb Incorporated, Rochester, NY, USA; Pred Forte ${ }^{\circledR}$, Allergan, Inc., Irvine, CA, USA.

prednisolone acetate, respectively. Drug concentrations for the three formulations determined on samples which were not shaken prior to expression of the 2-drop sample are shown in Figure 2. LE gel drug concentrations were again consistent with the declared concentrations across all samples analyzed on all dosing days at all dosing time points. The overall mean (SD) percent declared concentration for LE samples was $102 \%(1.92 \%)$. The dose uniformity of Pred Forte and the generic prednisolone acetate $1 \%$ drops was highly variable across all the samples analyzed, when not shaken prior to expressing drops. The relative SDs for the percent declared concentrations were $18.5 \%$ for Pred Forte and $22.0 \%$ for the generic prednisolone acetate suspension. In addition, mean drug concentrations in the prednisolone acetate drops were significantly below the declared concentration at the beginning of the 14-day simulated dosing regimen, increased over time, and were significantly above the declared concentration near the end of the 14-day simulated dosing regimen. The prednisolone acetate drops provided, on average, only $75 \%$ of the declared drug concentration (range of $72 \%-87 \%$ for Pred Forte and $71 \%-81 \%$ for the generic prednisolone acetate) at the beginning of the simulated dosing regimen. This increased over time to an average of approximately $10 \%$ above the declared concentration on Day 14 (range of $77 \%-121 \%$ for Pred Forte and $73 \%-132 \%$ for the generic prednisolone acetate).

Results of the additional sedimentation experiment are presented in Figures 3 and 4. Figure 3 presents photographs of LE ophthalmic gel, Pred Forte, and generic prednisolone acetate taken before and after centrifugation at 1,000 rpm for 24 hours. Before centrifugation, all formulation samples were opaque, indicating that their drug particles were well dispersed. After 24 hours of centrifugation, the LE ophthalmic gel sample was still opaque and apparently well dispersed, while the drug particles in Pred Forte and generic prednisolone acetate had sedimented, leaving most of the sample translucent. Figure 4 presents the percent transmittance area
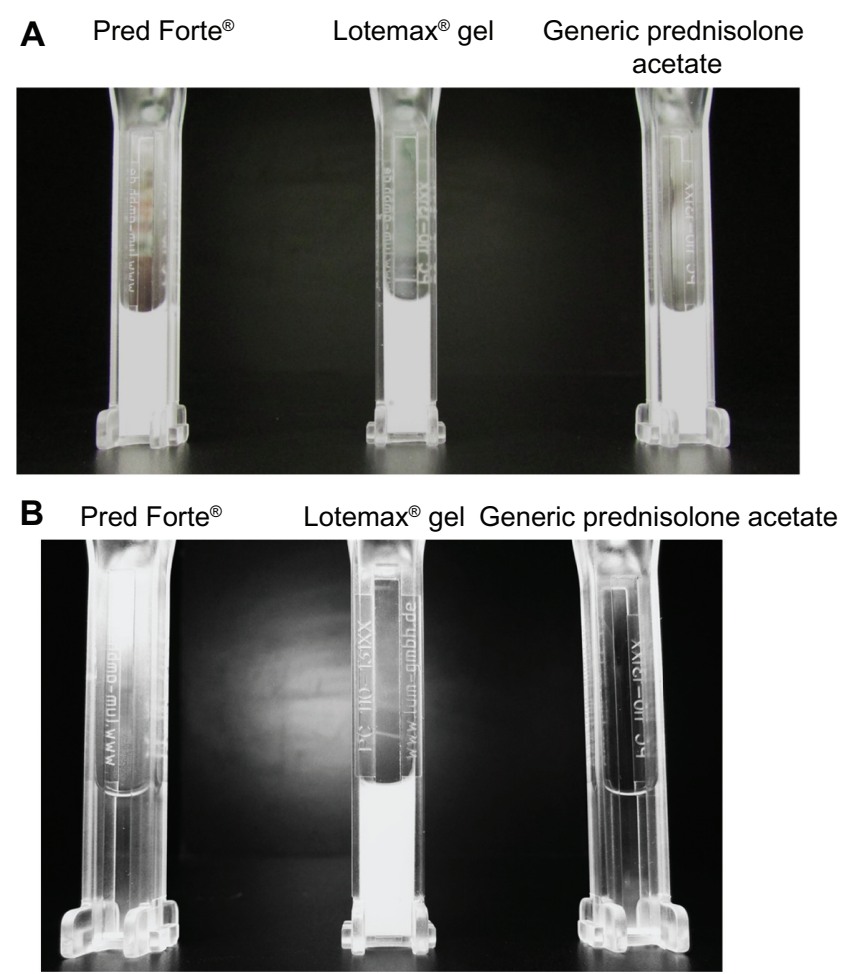

Figure 3 Sedimentation of Lotemax ${ }^{\circledast}$ ophthalmic gel $0.5 \%$, Pred Forte ${ }^{\circledR}$, and the generic prednisolone acetate suspension $1 \%$, following centrifugation at $1,000 \mathrm{rpm}$ (I20×g) for 24 hours.

Notes: The images are photographs of Lotemax ${ }^{\circledR}$ gel, Pred Forte ${ }^{\circledR}$, and generic prednisolone acetate before $(\mathbf{A})$ and after $(\mathbf{B})$ centrifugation. Lotemax ${ }^{\circledR}$, Bausch \& Lomb Incorporated, Rochester, NY, USA; Pred Forte ${ }^{\circledR}$, Allergan, Inc., Irvine, CA, USA. 


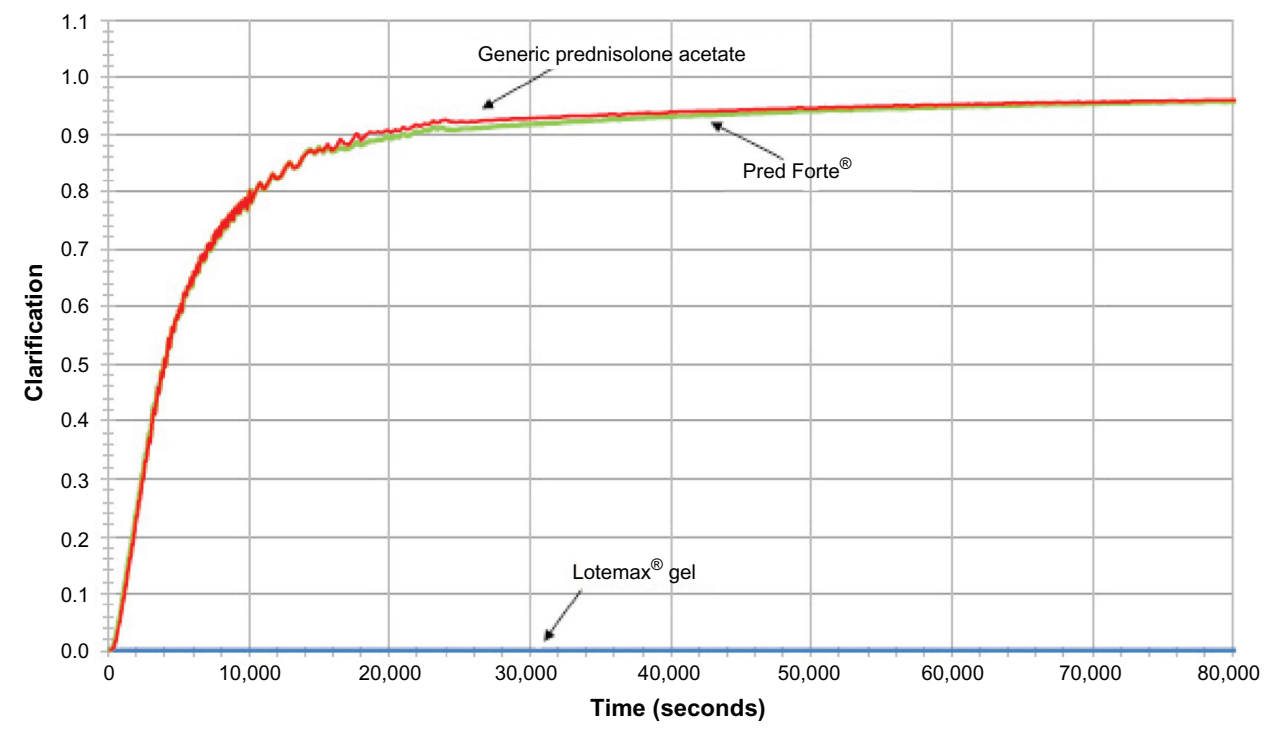

Figure 4 Kinetics of the sedimentation of Lotemax ${ }^{\circledR}$ ophthalmic gel $0.5 \%$, Pred Forte ${ }^{\circledR}$, and generic prednisolone acetate suspension I\%, at I,000 rpm (I20X g). Note: Clarification $=$ normalized integral of the percent transmittance. Lotemax ${ }^{\circledR}$, Bausch \& Lomb Incorporated, Rochester, NY, USA; Pred Forte ${ }^{\circledR}$, Allergan, Inc., Irvine, CA, USA.

under the curve (AUC) as a function of centrifugation time. LE gel showed no indication of settling during the entire course of the 24-hour centrifugation experiment, whereas both prednisolone acetate suspensions settled completely by 6 hours.

\section{Discussion}

LE ophthalmic gel $0.5 \%$ has been shown to be safe and effective for the treatment of pain and inflammation following cataract surgery in two multicenter, double-masked, randomized, vehicle-controlled clinical studies. ${ }^{5,6}$ Coffey and Davio previously described the unique rheologic properties of LE ophthalmic gel $0.5 \%$, specifically, the semisolid properties of the product at the resting state and the fluid properties of the product under mild shear conditions. ${ }^{2}$ These properties resulted in well-suspended particles that did not settle over a 16-month period of stability testing (upright storage at $25^{\circ} \mathrm{C}$ and $40^{\circ} \mathrm{C}$ ), with drop uniformity ranging from $100.4 \%-107.6 \%$ of the declared concentration. ${ }^{2}$ The results of the current studies further demonstrate an advantage of the nonsettling LE ophthalmic gel formulation: drug concentrations of LE in expressed drops were uniform, with declared drug concentrations that were within one or two percentage points of the declared concentration over the entire 2-week simulated dosing regimen and an overall relative SD of less than $2 \%$, regardless of whether the formulation was shaken or not shaken prior to expressing the drop. In contrast, conventional suspensions, represented in this study by both branded (Pred Forte) and generic prednisolone acetate 1\%, required shaking prior to dosing in order to resuspend the settled drug particles and obtain consistent dose uniformity. The nonsettling characteristic of LE ophthalmic gel was further confirmed under accelerated sedimentation conditions: no drug particle sedimentation was observed for LE gel even after centrifugation at 1,000 rpm for 24 hours, whereas the prednisolone acetate drug particles settled out of solution within 6 hours.

Results for the prednisolone acetate suspensions in this study differed from the results obtained by Stringer and Bryant in their study on dose uniformity of corticosteroid preparations as a function of shaking. ${ }^{7}$ In our study, uniform prednisolone acetate-drop concentrations close to declared concentrations were achieved by manually shaking Pred Forte and a generic prednisolone acetate suspension for 5 seconds. In the cited study, which utilized the same dosing regimen, the dose uniformity of the generic prednisolone acetate suspension was highly variable even with 5 seconds of shaking and was significantly below $(\sim 30 \%)$ the declared drug concentration for almost the full duration of the simulated dosing regimen. However, in agreement with our study, the dose uniformity of the branded prednisolone acetate (Pred Forte) was generally consistent with the declared drug concentration following 5 seconds of shaking, with the exception of the drug concentrations in the initial drops extruded, which were several fold higher than the declared concentration (312\%). The differences between our data and those obtained by Stringer and Bryant could be due to differences in the generic product tested and/or differences in the manner in which the products were shaken. 
The dosing of ophthalmic suspensions can be quite variable as a result of patient shaking behavior. Apt et al determined that only 37 of 100 patients actually shook a dropper bottle prior to administering a dose of an ophthalmic suspension to the eyes of a manikin, in spite of clear label directions instructing that the product should be shaken. ${ }^{3}$ The authors calculated that those who did not shake the product would have administered $29 \%$ or less of the target concentration for the four corticosteroid suspensions tested. For those who did shake the product, the number of dropper bottle inversions varied widely. Diestelhorst et al further showed that there are differences in the intensity of product shaking within various patient populations. ${ }^{8}$ For example, elderly patients shake medications with less intensity than do young patients. Adding to such variability, most patients have no way of visually confirming that they have shaken the product sufficiently as containers are often opaque, white bottles.

Different suspension products are also quite variable with regard to their ease of resuspension. Using a pneumatic shaker at a shaking intensity of $200 \mathrm{~m}^{2} / \mathrm{s}^{3}$, Diestelhorst et al resuspended ophthalmic suspensions of dexamethasone, indomethacin, and prednisolone acetate; only the prednisolone acetate suspension showed an acceptable mean drop concentration, approaching the declared drug concentration, as well as a satisfactory coefficient of variation. ${ }^{8}$ Dexamethasone drop concentrations remained well below the declared concentration throughout the simulated dosing period, despite mechanical shaking. Indomethacin drop concentrations started below the declared concentration and gradually increased to concentrations above the declared concentration; this is similar to what we observed for unshaken prednisolone acetate, both branded and generic, in the current study.

Taken together, these data suggest that patient shaking behavior and ability to shake containers vigorously, combined with the inherent variability in the ease of resuspension between products, can result in highly variable dosing for topical ophthalmic suspensions. As demonstrated in this study and others, this variability may take the form of low doses initially but higher concentrations toward the end of treatment. Indeed, in the current study, in the absence of shaking, the mean drug concentrations for both the branded and generic prednisolone acetate formulations were as much as $25 \%$ below the declared concentration for drops expressed at the beginning of the 2 -week dosing regimen and $10 \%$ above the declared concentration for drops expressed near the end of the dosing regimen, with large variability around the mean. Low doses at the beginning of treatment may be problematic, in that drug concentrations may not reach the required levels for clinical efficacy. Higher doses of a corticosteroid at the end of treatment could be problematic due to the potential for adverse ocular effects, such as elevated intraocular pressure (IOP). ${ }^{9}{ }^{10}$ Thus, patients who do not fully resuspend their prednisolone acetate suspensions by shaking prior to dosing may be at greater risk of elevated IOP near the end of the treatment period, when the drug concentrations are expected to be higher. Although the clinical impact of dose variability was not evaluated in our study, Roberts and Nelson previously demonstrated significantly better visual acuity at 1 month in postoperative eyes with severe inflammation that were treated with Pred Forte compared with those treated with a generic prednisolone acetate formulation, a difference the author attributed to the greater difficulty in resuspending the generic formulation. ${ }^{11,12}$ Other examples of differences in clinical efficacy due to dose variability were reviewed by Zore et al in the context of generic ophthalmic medications. ${ }^{13}$

Because it is nonsettling, clinicians may be assured that consistent dose uniformity and the therapeutic efficacy of LE ophthalmic gel $0.5 \%$ will be delivered without the requirement to shake the bottle prior to each use and that safety concerns related to potentially hyperconcentrated dosages near the end of treatment will not occur. In this regard, clinical studies with LE gel in the treatment of postoperative inflammation and pain have demonstrated a significant difference in the proportion of patients with complete resolution of protein flare and grade 0 (no) pain at the first on-treatment follow-up visit, namely, day 3 of dosing: adverse events were few and occurred less frequency in patients treated with LE ophthalmic gel compared with vehicle-treated patients. ${ }^{7,8}$ Moreover, LE itself, a C-20 ester corticosteroid, has been shown to have a lower potential to elevate IOP compared with other corticosteroids. ${ }^{14,15,16}$ This is due to structural modifications to the drug molecule that facilitate rapid and predictable metabolism (to inactive metabolites) of any LE not bound to the glucocorticoid receptor, resulting in a greater therapeutic index compared with other C-20 ketone corticosteroids. ${ }^{9}$ Another potential adverse event associated with the use of corticosteroids is the formation of cataracts in phakic individuals. In addition to its rapid metabolism, as a $\mathrm{C}-20$ ester rather than $\mathrm{C}-20$ ketone corticosteroid, LE is unable to form Schiff base intermediates with lens proteins, considered a first step in cataractogenesis, although other mechanisms of cataract formation may exist. ${ }^{17}$

In conclusion, LE ophthalmic gel $0.5 \%$ provided consistent dose uniformity whether or not the bottle was shaken 
prior to dispensing. The greater intrinsic safety profile of LE itself, along with the higher consistency of dosing of the LE ophthalmic gel, provides a significant advantage to patients compared with conventional corticosteroid suspensions.

\section{Acknowledgments}

The authors wish to thank Drs Heleen DeCory, Timothy Comstock, and Sanjay Malieckal for their careful reviews of this manuscript and their helpful suggestions.

\section{Disclosure}

The authors are employees of Bausch and Lomb, Inc. Editorial support, provided by Churchill Communications, was funded by Bausch and Lomb. The authors report no other conflicts of interest.

\section{References}

1. Lotemax ${ }^{\circledR}$ (loteprednol etabonate ophthalmic ointment $0.5 \%$ ) [US prescribing information]. Tampa, FL: Bausch and Lomb, Inc; 2012.

2. Coffey MJ, Davio SR. Viscoelastic and sedimentation characterization of loteprednol etabonate ophthalmic gel, $0.5 \%$. Poster presented at: the Association for Research in Vision and Ophthalmology (ARVO); May 6-10, 2012; Fort Lauderdale, FL.

3. Apt L, Henrick A, Silverman LM. Patient compliance with use of topical ophthalmic corticosteroid suspensions. Am J Ophthalmol. 1979;87(2): 210-214.

4. Pred Forte ${ }^{\circledR}$ (prednisolone acetate ophthalmic suspension 1\%) [US prescribing information]. Irvine, CA: Allergan, Inc; 2004.
5. Rajpal RK, Roel L, Siou-Mermet R, Erb T. Efficacy and safety of loteprednol etabonate $0.5 \%$ gel in the treatment of ocular inflammation and pain after cataract surgery. J Cataract Refract Surg. 2013;39(2):158-167.

6. Fong R, Leitritz M, Siou-Mermet R, Erb T. Loteprednol etabonate gel $0.5 \%$ for postoperative pain and inflammation after cataract surgery: results of a multicenter trial. Clin Ophthalmol. 2012;6:1113-1124.

7. Stringer W, Bryant R. Dose uniformity of topical corticosteroid preparations: difluprednate ophthalmic emulsion $0.05 \%$ versus branded and generic prednisolone acetate ophthalmic suspension 1\%. Clin Ophthalmol. 2010;4:1119-1124.

8. Diestelhorst M, Kwon KA, Süverkrup R. Dose uniformity of ophthalmic suspensions. J Cataract Refract Surg. 1998;24(5):672-677.

9. Bodor N, Buchwald P. Ophthalmic drug design based on the metabolic activity of the eye: soft drugs and chemical delivery systems. AAPS J. 2005;7(4):E820-E833.

10. McGhee CN, Dean S, Danesh-Meyer H. Locally administered ocular corticosteroids: benefits and risks. Drug Saf. 2002;25(1):33-55.

11. Roberts CW, Nelson PL. Comparative analysis of prednisolone acetate suspensions. J Ocul Pharmacol Ther. 2007;23(2):182-187.

12. Roberts CW. A comparison of branded to generic prednisolone acetate for control of postoperative inflammation. Invest Ophthalmol Vis Sci. 2000;41:S150.

13. Zore M, Harris A, Tobe LA, et al. Generic medications in ophthalmology. Br J Ophthalmol. 2013;97(3):253-257.

14. Pleyer U, Ursell PG, Rama P. Intraocular pressure effects of common topical steroids for post-cataract inflammation: are they all the same? Ophthalmol Ther. Epub September 17, 2013.

15. Comstock TL, Decory HH. Advances in corticosteroid therapy for ocular inflammation: loteprednol etabonate. Int $J$ Inflam. 2012;2012:789623.

16. Amon M, Busin M. Loteprednol etabonate ophthalmic suspension $0.5 \%$ : efficacy and safety for postoperative anti-inflammatory use. Int Ophthalmol. 2012;32(5):507-517.

17. Manabe S, Bucala R, Cerami A. Nonenzymatic addition of glucocorticoids to lens proteins in steroid-induced cataracts. $J$ Clin Invest. 1984;74(5):1803-1810.
Clinical Ophthalmology

\section{Publish your work in this journal}

Clinical Ophthalmology is an international, peer-reviewed journal covering all subspecialties within ophthalmology. Key topics include: Optometry; Visual science; Pharmacology and drug therapy in eye diseases; Basic Sciences; Primary and Secondary eye care; Patient Safety and Quality of Care Improvements. This journal is indexed on

\section{Dovepress}

PubMed Central and CAS, and is the official journal of The Society of Clinical Ophthalmology (SCO). The manuscript management system is completely online and includes a very quick and fair peer-review system, which is all easy to use. Visit http://www.dovepress.com/ testimonials.php to read real quotes from published authors. 